\title{
How Do We Enhance Oncology Nurses' Caring Utilizing Resilience And Professional Quality of Life?: A cross-Sectional Descriptive Study
}

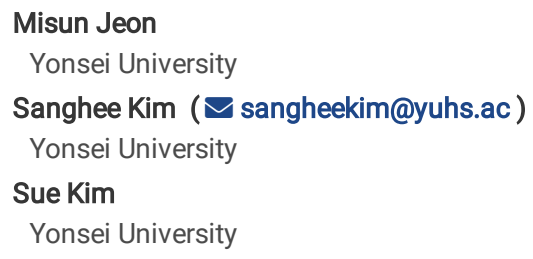

\section{Research Article}

Keywords: oncology nurse, resilience, quality of life, caring, cancer, compassion satisfaction, burnout, secondary traumatic stress

Posted Date: January 13th, 2022

DOI: https://doi.org/10.21203/rs.3.rs-1236760/v1

License: (c) (i) This work is licensed under a Creative Commons Attribution 4.0 International License. Read Full License 


\section{Abstract}

Background: Degree of caring behavior in oncology nurses is a crucial factor in caring for patients with cancer. It is important to understand factors related to oncology nurses' caring including their own resilience and professional quality of life. The purpose of this study was to investigate oncology nurses' resilience, professional quality of life, and caring.

Methods: A cross-sectional descriptive correlational study was conducted on 107 oncology nurses using self-report questionnaires. Data analysis included descriptive statistics, Pearson's correlations, and stepwise multiple regression to examine factors affecting oncology nurses' degree of caring behavior, using SPSS/WIN 20.0 program (IBM).

Result: Oncology nurses presented a low level of resilience and degree of caring behavior, a high level of compassion satisfaction, burnout, and secondary traumatic stress. There was a statistically significant relationship between degree of caring behavior, resilience $(r=.43, p<.001)$, compassion satisfaction ( $r$ $=.51, p<.001)$, and burnout $(r=-.42, p<.001)$ as well as between secondary traumatic stress and burnout $(r=.34, p<.001)$. Factors affecting oncology nurses' degree of caring behavior were compassion satisfaction and education level.

Conclusions: This study demonstrated that oncology nurses' degree of caring behavior relates to professional quality of life, and education. This association suggests several implications for nursing practice to encourage holistic nursing. It will be necessary to study the factors affecting nurses' compassion satisfaction, and to try to promote compassion satisfaction according to the study.

\section{Background}

Cancer patients invariably undergo a complex treatment process, starting with a diagnosis followed by a combination of surgery, chemotherapy, and radiation therapy, thereby experiencing several side effects. In the process, cancer patients and their families require increased nursing care, as they experience a high degree of uncertainty [1].

Oncology nurses' caring for cancer patients and their families, at the forefront, frequently encounter stressful experiences such as patients' pain, medical limitations, and family distress. Oncology nurses particularly feel fear of death, guilt, and burden, from caring for cancer patients. Performing special tasks, such as end-of-life nursing and administering chemotherapy to cancer patients, result in feeling lower job satisfaction, compassion fatigue, and burnout among oncology nurses, compared with nurses in general wards [2-4], as they experience higher job stress [5]. Studies showed that oncology nurses often experiece high job stress. Job stress includes psychological aspects such as anxiety, depression, insomnia, burnout, and decreased quality of life [6-8], physical aspects such as muscle tension, tachycardia, obesity, and health status [5], low job satisfaction [8] and a negative impact on organizational aspects such as the impulse to resign [9]. Oncology nurses often complain about the compassion fatigue and burnout. It has been reported as related to low job satisfaction in addition to job stress [10]. Incidentally, low job satisfaction decreases overall life satisfaction [11]. In other words, the burnout, compassion fatigue, low job satisfaction, and high job stress experienced by oncology nurses can negatively impact caring for patients and lower nurses' personal life satisfaction $[12,13]$. However, these negative outcomes, such as job stress or burnout, would generally not be expected to exist for all oncology nurses due to individual differences in coping with stress [14]

One of the distinct abilities in coping with stress is resilience. Resilience is defined as the capacity to recover from external adversities, such as job stress. Highly resilient nurses overcome difficulties by actively utilizing their internal and external resources [15]. High resilience has been reported as leading to a sense of well-being, high professional quality of life, and job enthusiasm, as well as low burnout and job dissatisfaction, in the results of several studies [16, 17].

Resilience and professional quality of life are related to job satisfaction, job stress, and burnout, which affect nursing outcomes, including care [18]. Furthermore, emotional, spiritual, and physical care are fundamental attributes of nursing practice. Additionally, a high degree of care received from nurses directly impacts patient outcomes [19].

Several studies on resilience and professional quality of life related to caring have been conducted on nurses in general wards. From these studies, we estimated the relationship between the level of resilience and professional quality of life, according to the general characteristics of the nurses. Although nurses for oncology patients perform more individual care than nurses working in general wards [20], there is limited research on their job characteristics. It can be predicted that job characteristics will cause high job stress and burnout. Nevertheless, there are very few studies researching the association between resilience and professional quality of life related to caring, specific to oncology nurses

Consequently, in this study, we attempted to explore the factors that affect the oncology nurses' degree of caring behavior, which can further improve the quality of care for cancer patients. We specifically examined resilience, professional quality of life, and degree of caring behavior of oncology nurses to provide intervention necessary to improve care.

Specifically, this study aimed to 1) assess nurses' resilience, professional quality of life, and degree of caring behavior 2) analyze the differences in levels of resilience, professional quality of life, and degree of caring behavior based on their general characteristics 3 ) identify the relationship between resilience, professional life quality, and degree of caring behavior was confirmed 4) find the factors that influence the degree of caring behavior of oncology nurses.

\section{Methods}

\section{Study design and participants}


This study is a cross-sectional descriptive correlational study conducted with the oncology nurses working at a tertiary hospital located in Seoul, Korea. Participants were oncology nurses who received information about the study through poster advertisements at patient care unit and informational brochures distributed to their work mailboxes. Those eligible for the study were all nurses who worked cancer patients care unit in the oncology ward, outpatient chemotherapy treatment center, and hematopoietic stem cell transplant ward. Participants also had to be willing. Interested participants initially were interviewed to ensure their understanding of the study and its anticipated benefits and risks. During the survey, if participants want to stop, they can stop at any time. After the participants expressed their intention to participate, researchers obtained participants' written informed consent

In this study, the G*Power 3.1 program was used to estimate the sample size, with the significance level set at .05, a power of .80, and an effect size of .15, a median effect size of regression, and with 6 independent variables (resilience, professional quality of life's three subdomains, education, job satisfaction) based on the literature. This resulted in a minimum sample size of 98 . Considering a dropout rate of $12 \%$ [5] the target sample size was determined at 118 nurses. This questionnaire was distributed among 118 nurses, from which 111 responses (recovery rate $96.52 \%$ ) were collected, and 107 were used in the final analysis, excluding four incomplete responses.

We used a structured self-report questionnaire consisting of 89 questions, 25 items for resilience, 30 for the professional quality of life, 20 for the degree of care, and 14 for general characteristics with the approval of the original author and the translator. The total time required to complete the questionnaire was $20 \mathrm{~min}$.

\section{1) Resilience}

Connor-Davidson Resilience Scale (CD-RISC) developed by Conor and Davidson [21]. The Korean Connor-Davidson Resilience Scale (K-CD-RISC), adapted and validated by Baek Hyun-sook [22], was used to measure resilience in oncology nurses. This tool consists of a total of 25 questions and five subdomains; toughness, perseverance, optimism, support, and spirituality, measured on a 5 -point Likert scale ( 0 not at all, 4 very so). And the tool's total score ranges from 0 to 100. Higher scores indicate higher levels of resilience. Regarding the reliability of the tool, Cronbach's a was .91 in Baek's study [22] and .92 in the present study.

\section{2) Professional quality of life}

Professional Quality of Life Scale: Compassion Satisfaction and Compassion Fatigue Version 5 developed by Stamm [23]. This tool consists of a total of 30 questions, 10 questions for each of the three subdomains: compassion satisfaction, burnout, and secondary traumatic stress, measured on a 5-point Likert scale ranging from 1 'not at all' to 5 'very often'. The total score of each sub-factor is independently evaluated without using the total score of the 30 questions, for a possible range of scores from 10 to 50 . Additionally, to determine the high-risk group with low professional quality of life, the total score of each subfactor is standardized with a $Z$ score of 50 and a variance of 10 , and then the standardized score is compared with the average of the population. A percentile range of $75 \%$ or higher is categorized as high; $25-75 \%$ as medium; and $25 \%$ or lesser as low [22]. Regarding the reliability of the tool, Cronbach's a in Stamm's study [23] was .88 for compassion satisfaction, .75 for burnout, and .81 for secondary traumatic stress. In this study, Cronbach's a was .91 for compassion satisfaction, .78 for burnout, and .75 for secondary traumatic stress.

\section{3) Caring}

The Care Factor Survey-Care Provider Version developed by Watson and Nelson [24]. It was subsequently translated and discussed by a bilingual expert through a committee method [25]. We used this tool to measure degree of caring behavior which are humanistic practice, faith in decision making, instilling faith and hope, learning and teaching, spiritual beliefs and practices, holistic care, helping and trusting relationships, creating a therapeutic environment, promoting emotional expression, and accepting miracles. Each of these ten perception factors for caring consisted of two questions, measured on a 7-point Likert scale ranging from 1 'disagree' to 7 'strongly agree'. The total score ranges from 20 to 140; the higher the score, the higher the level of care. Regarding the reliability, Cronbach's a was .92 in Johnson's study [24] and .94 in the present study.

\section{Procedures}

Data collection was done from May 18 to 24,2015 . We provided written information, including the purpose and nature of this study, anonymity of data, guaranteed confidentiality, and the option to withdraw from the study at any time, to potential nurses and obtained their signed consent to participate. Those who read the explanation and agreed to participate in the study completed a self-report questionnaire.

\section{Data analysis}

The collected data were analysed using IBM SPSS/WIN 20.0 statistical program. Using an independent t-test, one-way ANOVA, and the Scheffé post-hoc test, we analysed the difference in resilience, professional quality of life, and degree of caring behavior, according to the general characteristics of the nurses. Then, we calculated Pearson's correlation coefficients to investigate the relationship between the nurses' resilience, professional quality of life, and degree of caring behavior. Lastly, stepwise regression was performed to identify the factors affecting the degree of caring behavior of the nurses.

\section{Results}

Participants' general characteristics

All study participants consisted of women, nurses, whose average age was $28.79 \pm 5.53$ years. Of these, $67.3 \%$ were under 30 years old, and $75.7 \%$ were married. Among all, $53.5 \%$ graduated from a four-year university, $63.6 \%$ reported religiosity, and $95.3 \%$ indicated having someone who can advise or help them. Among their family and acquaintances, $48.6 \%$ of the nurses did not have cancer patients. Clinically, most experienced nurses had 7 years or more (36.4\%), while the average clinical experience was $6.31 \pm 5.67$ years. The most clinical experience related to cancer patients was also over 7 years ( $29 \%)$, with an 
average of $4.96 \pm 4.54$ years. As for the position held, $56.1 \%$ of them were more than charge nurses. Regarding their working department, the internal surgery ward was common to $82.2 \%$, and $78.5 \%$ worked in three shifts. The highest job satisfaction reported was by $47.7 \%$, with $4-6$ points in the middle, and an average of $5.84 \pm 1.78$ points out of 10 points, and $59.8 \%$ of them were able to take a break on the desired holiday. (Table 1 ).

Table 1

General Characteristics of the Participants ( $N=107)$

\begin{tabular}{|c|c|c|c|}
\hline Characteristics & Categories & $N(\%)$ & Mean $\pm S D$ \\
\hline \multirow[t]{3}{*}{ Age(year) } & $\sim<30$ & 72(67.3) & $28.79 \pm 5.53$ \\
\hline & $30 \sim<34$ & $17(15.9)$ & \\
\hline & $\geq 35$ & $18(12.1)$ & \\
\hline \multirow[t]{2}{*}{ Marital status } & Never married & $81(75.7)$ & \\
\hline & Married & $26(24.3)$ & \\
\hline \multirow[t]{3}{*}{ Education } & Diploma & $37(34.6)$ & \\
\hline & BSN & $57(53.3)$ & \\
\hline & Higher than Graduate program & 13(12.1) & \\
\hline \multirow[t]{4}{*}{ Religion } & Christian & $26(24.3)$ & \\
\hline & Catholic & $5(4.7)$ & \\
\hline & Buddhist & $8(7.5)$ & \\
\hline & No religion & $68(63.6)$ & \\
\hline \multirow{4}{*}{$\begin{array}{l}\text { Clinical experience } \\
\text { (year) }\end{array}$} & $<1$ & 19(17.8) & $6.31 \pm 5.67$ \\
\hline & $1 \sim<3$ & 18(16.8) & \\
\hline & $3 \sim<7$ & $31(29.0)$ & \\
\hline & $\geq 7$ & $39(36.4)$ & \\
\hline \multirow{4}{*}{$\begin{array}{l}\text { Clinical experience with cancer nursing } \\
\text { (year) }\end{array}$} & $<1$ & $26(24.3)$ & $4.96 \pm 4.54$ \\
\hline & $1 \sim<3$ & $23(21.5)$ & \\
\hline & $3 \sim<7$ & $27(25.2)$ & \\
\hline & $\geq 7$ & $31(29.0)$ & \\
\hline \multirow[t]{2}{*}{ Shift work } & Three shifts & $84(78.5)$ & \\
\hline & Daytime & $23(21.5)$ & \\
\hline \multirow[t]{2}{*}{ Clinical position } & Staff nurse & $47(43.9)$ & \\
\hline & Charge ${ }^{\star} /$ Head nurse & $60(56.1)$ & \\
\hline \multirow[t]{2}{*}{ Workplace } & General medicine-surgical ward & $88(82.2)$ & \\
\hline & Outpatient clinic/Floating & 19(17.8) & \\
\hline \multirow[t]{2}{*}{ Education related to cancer nursing } & Yes & $74(69.2)$ & \\
\hline & No & $33(30.8)$ & \\
\hline \multirow[t]{2}{*}{ Can take a day off at any time } & Yes & $64(59.8)$ & \\
\hline & No & $43(40.2)$ & \\
\hline \multirow[t]{3}{*}{ Cancer patients experience with family or friends } & Family & $37(34.6)$ & \\
\hline & Friends & 18(16.8) & \\
\hline & None & $52(48.6)$ & \\
\hline \multirow[t]{2}{*}{ Having a helper or counsellor nearby } & Yes & 102(95.3) & \\
\hline & No & $5(4.7)$ & \\
\hline \multirow[t]{3}{*}{ Job satisfaction } & $0 \sim 3($ Low) & $9(8.4)$ & $5.84 \pm 1.78$ \\
\hline & 4 6(Medium) & $51(47.7)$ & \\
\hline & 7 10(High) & $47(43.9)$ & \\
\hline
\end{tabular}


The nurses' total resilience was slighter higher than midpoint, i.e., $57.81 \pm 11.49$ out of 100 points, and the average score was $2.31 \pm 0.46$ out of 4 points. And nurses' compassion satisfaction as one of the professional quality of life (average score of $3.36 \pm 0.5$ points $/ 5$ points) and degree of caring behavior (average $4.76 \pm 0.67$ points/7 points) were higher than midpoint. (Table 2).

Table 2

Score of Resilience, Professional Quality of Life and Caring ( $N=107)$

\begin{tabular}{|llllll|}
\hline Variable & Number of items & Min & Max & Mean \pm SD & Average mean \pm SD \\
\hline Resilience & 25 & 31 & 83 & $57.81 \pm 11.49$ & $2.31 \pm 0.46 / 4.00$ \\
\hline Professional quality of life & & & & & \\
\hline \multicolumn{1}{c}{ Compassion satisfaction } & 10 & 16 & 45 & $33.58 \pm 5.66$ & $3.36 \pm 0.57 / 5.00$ \\
\hline Burnout & 10 & 16 & 41 & $28.03 \pm 4.95$ & $2.80 \pm 0.50 / 5.00$ \\
\hline Secondary traumatic stress & 10 & 15 & 42 & $27.51 \pm 4.40$ & $2.75 \pm 0.44 / 5.00$ \\
\hline Caring & 20 & 65 & 135 & $95.20 \pm 13.39$ & $4.76 \pm 0.67 / 7.00$ \\
\hline
\end{tabular}

As a result of the group analysis of professional quality of life, the median group showed the most compassion satisfaction, burnout, and secondary traumatic stress, with $43 \%, 45.8 \%$, and $60.7 \%$, respectively. Furthermore, $75.7 \%$ of the nurses indicated moderate or higher levels of burnout, $83.1 \%$ indicated moderate or higher secondary traumatic stress, and $26.2 \%$ indicated high compassion satisfaction (Table 3). Additionally, in the transformed z-values to identify high-risk groups with low professional quality of life, we found that $5.61 \%$ belonged to the high-risk group. In this group, the average of resilience $(42.83+7.14$ points) and degree of caring behavior ( $87.67+11.34$ points) were lower levels than the overall average.

Table 3

Level of Professional Quality of Life $(\mathrm{N}=107)$

\begin{tabular}{|llll|}
\hline Variable & \multicolumn{2}{l|}{$\begin{array}{l}\text { Professional quality of life } \\
\text { N (\%) }\end{array}$} \\
\cline { 2 - 4 } & Compassion satisfaction & Burnout & Secondary traumatic stress \\
\hline High (>75\%) & $28(26.2)$ & $32(29.9)$ & $24(22.4)$ \\
\hline Medium (25 75\%) & $46(43)$ & $49(45.8)$ & $65(60.7)$ \\
\hline Low (<25\%) & $33(30.8)$ & $26(24.3)$ & $18(16.8)$ \\
\hline
\end{tabular}

Differences in resilience, professional quality of life, and caring

Nurses over 35 years old than 34 years were higher levels of resilience $(F=4.26, p=.017)$ and degree of caring behavior $(F=6.79, p=.002)$. And they were lower levels of burnout $(F=5.42, p=.006)$. Married nurses than unmarried showed higher levels of resilience $(t=-3.01$, $p=.003)$, compassion satisfaction ( $t=-2.27$, $\mathrm{p}=.025)$, and degree of caring behavior $(t=-2.26, p=.026)$. And they were lower levels of burnout $(t=4.43, p<.001)$. If job satisfaction was higher, nurses' resilience $(F=46.59, p<.001)$, compassion satisfaction $(t=-2.27, p=.025)$, and degree of caring behavior $(F=17.39, p<.001)$ were significantly higher. And higher job satisfaction than lower was lower burnout $(F=45.18, p<.001)$. When others were available to consult with, they were higher compassion satisfaction $(t=3.10, p=.002)$ and lower burnout $(t=-2.35, p=.021)$. The burnout was higher for those with the total clinical experience between 1 and 3 years than for those with 7 years or more $(F=3.45, p=.019)$ and in the staff nurses than the charge nurses and head nurses $(t=2.15$, $p=.034)$. Secondary traumatic stress was higher in the case of the third shift than in the case of full-time jobs $(t=2.25, p=026)$, and when working in the ward than in the case of working for both outpatients and the ward $(t=2.52, p=.013)$. The degree of caring behavior for the charge nurses and head nurses was higher than that of the staff nurses ( $t=-2.09$, $p=.039$ ). Additianally degree of caring behavior was significantly higher in nurses who were enrolled in a graduate school or higher than in those who graduated from a vocational college or a four-year university $(F=6.34, p=.003)$ (Table 4). 
Table 4

Differences in Resilience, Professional Quality of Life and Caring by Participant's Demographic Characteristics (N=107)

\begin{tabular}{|c|c|c|c|c|c|c|c|c|c|c|c|}
\hline \multirow[t]{3}{*}{ Characters } & \multirow[t]{3}{*}{ Categories } & \multirow{2}{*}{\multicolumn{2}{|c|}{ Resilience }} & \multicolumn{6}{|c|}{ Professional quality of life } & \multirow{2}{*}{\multicolumn{2}{|c|}{ Caring }} \\
\hline & & & & \multicolumn{2}{|l|}{$\begin{array}{l}\text { Compassion } \\
\text { satisfaction }\end{array}$} & \multicolumn{2}{|l|}{ Burnout } & \multicolumn{2}{|c|}{$\begin{array}{l}\text { Secondary } \\
\text { traumatic stress }\end{array}$} & & \\
\hline & & $M \pm S D$ & $\begin{array}{l}\text { tor } \\
F(p)\end{array}$ & $M \pm S D$ & $t$ or $F(p)$ & $\mathrm{M} \pm \mathrm{SD}$ & $t$ or $F(p)$ & $\mathrm{M} \pm \mathrm{SD}$ & $\begin{array}{l}\text { tor } \\
F(p)\end{array}$ & $\mathrm{M} \pm \mathrm{SD}$ & $t$ \\
\hline \multirow[t]{3}{*}{ Age(year) } & $\sim<27$ & $57.33 \pm 11.90$ & $4.26^{\star}$ & $32.96 \pm 6.4$ & 1.76 & $29.08 \pm 5.13$ & $5.42 * \star$ & $27.96 \pm 5.00$ & 1.05 & $92.71 \pm 11.75$ & 6 \\
\hline & $27 \sim<34$ & $55.86 \pm 10.43$ & $(.017)$ & $33.52 \pm 4.92$ & $(.177)$ & $27.90 \pm 4.41$ & $(.006)$ & $27.43 \pm 3.95$ & $(.354)$ & $94.60 \pm 14.81$ & (. \\
\hline & $\geq 35$ & $66.08 \pm 10.22$ & & $36.23 \pm 3.5$ & & $24.23 \pm 4.21$ & & $26.00 \pm 2.77$ & & $107.15 \pm 8.05$ & \\
\hline \multirow{2}{*}{$\begin{array}{l}\text { Marital } \\
\text { status }\end{array}$} & Never married & $55.99 \pm 11.34$ & $-3.01 * \star$ & $32.89 \pm 5.82$ & $-2.27 *$ & $29.14 \pm 4.58$ & $4.431^{\star \star \star}$ & $27.86 \pm 4.57$ & 1.46 & $93.58 \pm 12.19$ & -2 \\
\hline & Married & $63.50 \pm 10.21$ & $(.003)$ & $35.73 \pm 4.62$ & $(.025)$ & $24.58 \pm 4.52$ & $(<.001)$ & $26.42 \pm 3.68$ & $(.147)$ & $100.27 \pm 15.78$ & $($. \\
\hline \multirow[t]{3}{*}{ Education } & Diploma & $55.81 \pm 10.75$ & 1.53 & $33.46 \pm 5.85$ & .64 & $28.78 \pm 4.30$ & 2.36 & $27.30 \pm 4.93$ & .32 & $93.89 \pm 13.81$ & 6 \\
\hline & BSN & $58.12 \pm 11.46$ & $(.223)$ & $33.28 \pm 5.35$ & $(.532)$ & $28.14 \pm 5.02$ & $(.10)$ & $27.81 \pm 4.19$ & $(.729)$ & $93.37 \pm 12.96$ & $($. \\
\hline & $\begin{array}{l}\text { More than } \\
\text { Graduate } \\
\text { program }\end{array}$ & $62.15 \pm 13.20$ & & $35.23 \pm 6.58$ & & $25.38 \pm 5.82$ & & $26.85 \pm 3.85$ & & $107.00 \pm 7.44$ & \\
\hline \multirow[t]{4}{*}{ Religion } & Christian & $58.50 \pm 11.23$ & .16 & $34.31 \pm 4.02$ & .44 & $27.65 \pm 4.18$ & .13 & $27.65 \pm 5.21$ & .16 & $98.15 \pm 10.66$ & i \\
\hline & Catholic & $56.60 \pm 5.03$ & $(.922)$ & $34.00 \pm 5.29$ & $(.728)$ & $27.20 \pm 6.53$ & $(.943)$ & $28.20 \pm 2.28$ & $(.921)$ & $94.40 \pm 17.94$ & (. \\
\hline & Buddhist & $59.88 \pm 15.65$ & & $34.88 \pm 6.66$ & & $28.13 \pm 5.33$ & & $28.25 \pm 2.60$ & & $97.50 \pm 16.22$ & \\
\hline & No religion & $57.40 \pm 11.57$ & & $33.12 \pm 6.15$ & & $28.22 \pm 5.16$ & & $27.32 \pm 4.39$ & & $93.87 \pm 13.73$ & \\
\hline \multirow{4}{*}{$\begin{array}{l}\text { Clinical } \\
\text { experiences } \\
\text { (year) }\end{array}$} & $<1$ & $57.11 \pm 12.24$ & .61 & $33.21 \pm 5.27$ & .79 & $28.37 \pm 4.40$ & $3.45^{\star}$ & $27.79 \pm 4.53$ & .35 & $95.00 \pm 11.47$ & 2 \\
\hline & $1 \sim<3$ & $54.83 \pm 10.43$ & $(.612)$ & $31.89 \pm 7.37$ & $(.502)$ & $31.06 \pm 5.06$ & $(.019)$ & $28.06 \pm 6.07$ & $(.788)$ & $89.11 \pm 11.25$ & (. \\
\hline & $3 \sim<7$ & $58.35 \pm 11.32$ & & $33.90 \pm 6.07$ & & $27.71 \pm 4.89$ & & $27.74 \pm 4.22$ & & $93.74 \pm 14.55$ & \\
\hline & $\geq 7$ & $59.10 \pm 11.88$ & & $34.28 \pm 4.58$ & & $26.72 \pm 4.75$ & & $26.95 \pm 3.62$ & & $99.28 \pm 13.32$ & \\
\hline \multirow{4}{*}{$\begin{array}{l}\text { Clinical } \\
\text { experiences } \\
\text { with cancer } \\
\text { nursing } \\
\text { (year) }\end{array}$} & $<1$ & $55.85 \pm 11.94$ & .38 & $32.15 \pm 6.11$ & .85 & $28.69 \pm 5.04$ & 1.48 & $27.00 \pm 4.41$ & 1.08 & $94.73 \pm 11.92$ & 1 \\
\hline & $1 \sim<3$ & $57.87 \pm 1083$ & $(.767)$ & $34.57 \pm 6.49$ & $(.468)$ & $28.91 \pm 5.39$ & $(.224)$ & $28.04 \pm 5.83$ & $(.363)$ & $95.65 \pm 14.65$ & (. \\
\hline & $3 \sim<7$ & $58.22 \pm 11.45$ & & $33.56 \pm 5.73$ & & $28.41 \pm 4.47$ & & $28.52 \pm 3.61$ & & $90.78 \pm 13.94$ & \\
\hline & $\geq 7$ & $59.06 \pm 11.96$ & & $34.06 \pm 4.48$ & & $26.48 \pm 4.81$ & & $26.68 \pm 3.72$ & & $99.13 \pm 13.39$ & \\
\hline \multirow[t]{2}{*}{ Shift work } & Three shifts & $57.17 \pm 10.99$ & -.49 & $33.07 \pm 5.40$ & -1.34 & $28.71 \pm 4.48$ & 2.01 & $28.02 \pm 4.38$ & $2.25^{\star}$ & $93.79 \pm 13.83$ & -1 \\
\hline & Day-time & $58.55 \pm 13.13$ & $(.627)$ & $34.95 \pm 6.64$ & $(.185)$ & $25.80 \pm 6.11$ & $(.056)$ & $25.60 \pm 4.07$ & $(.026)$ & $98.65 \pm 9.85$ & (. \\
\hline \multirow{2}{*}{$\begin{array}{l}\text { Clinical } \\
\text { position }\end{array}$} & Staff nurse & $56.57 \pm 11.25$ & -.99 & $33.04 \pm 6.40$ & -.84 & $29.17 \pm 5.10$ & $2.15^{\star}$ & $27.89 \pm 5.29$ & .79 & $92.19 \pm 13.67$ & -2 \\
\hline & $\begin{array}{l}\text { Charge /Head } \\
\text { nurse }\end{array}$ & $58.78 \pm 11.68$ & $(.326)$ & $34.00 \pm 5.03$ & $(.402)$ & $27.13 \pm 4.68$ & $(.034)$ & $27.22 \pm 3.57$ & $(.432)$ & $97.57 \pm 12.79$ & (. \\
\hline \multirow[t]{3}{*}{ Workplace } & \multirow{3}{*}{$\begin{array}{l}\text { General } \\
\text { medicine- } \\
\text { surgical ward } \\
\text { Outpatient } \\
\text { clinic/Floating }\end{array}$} & $57.94 \pm 11.31$ & .25 & $33.43 \pm 5.48$ & -.58 & $28.49 \pm 4.54$ & 1.72 & $28.00 \pm 4.28$ & $2.52^{\star}$ & $94.69 \pm 13.85$ &.- \\
\hline & & $57.21 \pm 12.60$ & & $34.26 \pm 6.58$ & $(.564)$ & $25.89 \pm 6.24$ & $(.100)$ & $25.26 \pm 4.34$ & $(.013)$ & $97.58 \pm 10.99$ & $($. \\
\hline & & & & & & & & & & & \\
\hline \multirow{2}{*}{$\begin{array}{l}\text { Education } \\
\text { related } \\
\text { cancer } \\
\text { nursing }\end{array}$} & Yes & $59.10 \pm 11.37$ & 1.76 & $34.28 \pm 5.72$ & 1.95 & $27.59 \pm 5.26$ & -1.50 & $27.53 \pm 4.29$ & .05 & $96.07 \pm 13.92$ & 1 \\
\hline & No & $54.91 \pm 11.41$ & & $32.00 \pm 5.28$ & $(.054)$ & $29.00 \pm 4.07$ & $(.137)$ & $27.48 \pm 4.68$ & $(.964)$ & $93.27 \pm 12.09$ & $($. \\
\hline \multirow{2}{*}{$\begin{array}{l}\text { Can take a } \\
\text { day off at } \\
\text { any time }\end{array}$} & Yes & $59.50 \pm 11.10$ & 1.87 & $34.25 \pm 5.32$ & 1.50 & $27.59 \pm 4.72$ & -1.11 & $27.59 \pm 4.18$ & .23 & $96.05 \pm 14.03$ & .7 \\
\hline & No & $55.30 \pm 11.74$ & $(.064)$ & $32.58 \pm 6.07$ & $(.136)$ & $28.67 \pm 5.26$ & $(.270)$ & $27.39 \pm 4.75$ & $(.820)$ & $93.95 \pm 12.43$ & $($. \\
\hline \multirow{3}{*}{$\begin{array}{l}\text { Cancer } \\
\text { patients } \\
\text { experience } \\
\text { with family } \\
\text { or friends }\end{array}$} & Family & 56.3243 & $\begin{array}{l}.701 \\
(.496)\end{array}$ & 32.6216 & 1.17 & 28.7027 & .97 & 27.6757 & .05 & 94.6757 & 1 \\
\hline & Friends & 57.0000 & & 35.0556 & $(.315)$ & 26.7222 & $(.382)$ & 27.5556 & $(.954)$ & 99.5000 & (. \\
\hline & No & 59.1538 & & 33.7500 & - & 28.0000 & & 27.3846 & & 94.0962 & \\
\hline \multirow{2}{*}{$\begin{array}{l}\text { Having a } \\
\text { Helper or } \\
\text { counsellor } \\
\text { nearby }\end{array}$} & Yes & $58.14 \pm 11.22$ & \multirow{2}{*}{$\begin{array}{l}1.32 \\
(.189)\end{array}$} & $33.94 \pm 5.39$ & $3.10 * *$ & $27.78 \pm 4.83$ & $-2.35^{\star}$ & $27.41 \pm 4.45$ & -1.09 & $95.39 \pm 13.51$ & 1 \\
\hline & No & $51.20 \pm 16.27$ & & $26.20 \pm 6.61$ & $(.002)$ & $33.00 \pm 5.34$ & $(.021)$ & $29.60 \pm 2.61$ & $(.279)$ & $91.40 \pm 11.15$ & $($. \\
\hline
\end{tabular}




\begin{tabular}{|c|c|c|c|c|c|c|c|c|c|c|c|}
\hline \multirow[t]{3}{*}{ Characters } & \multirow[t]{3}{*}{ Categories } & \multirow{2}{*}{\multicolumn{2}{|c|}{ Resilience }} & \multicolumn{6}{|c|}{ Professional quality of life } & \multirow{2}{*}{\multicolumn{2}{|c|}{ Caring }} \\
\hline & & & & \multicolumn{2}{|l|}{$\begin{array}{l}\text { Compassion } \\
\text { satisfaction }\end{array}$} & \multicolumn{2}{|l|}{ Burnout } & \multicolumn{2}{|c|}{$\begin{array}{l}\text { Secondary } \\
\text { traumatic stress }\end{array}$} & & \\
\hline & & $\mathrm{M} \pm \mathrm{SD}$ & $\begin{array}{l}\text { tor } \\
F(p)\end{array}$ & $\mathrm{M} \pm \mathrm{SD}$ & $t$ or $F(p)$ & $\mathrm{M} \pm \mathrm{SD}$ & $t$ or $F(p)$ & $\mathrm{M} \pm \mathrm{SD}$ & $\begin{array}{l}\text { tor } \\
F(p)\end{array}$ & $\mathrm{M} \pm \mathrm{SD}$ & $\mathrm{t}$ \\
\hline \multirow{3}{*}{$\begin{array}{l}\text { Job } \\
\text { satisfaction }\end{array}$} & Low (0 3) & $42.56 \pm 6.82$ & \multirow{3}{*}{$\begin{array}{l}43.40 \\
(<.001)\end{array}$} & $23.78 \pm 4.58$ & $75.18^{\star \star \star}$ & $35.78 \pm 3.38$ & $45.18^{\star \star \star}$ & $27.11 \pm 4.31$ & .214 & $86.22 \pm 11.34$ & 1 \\
\hline & Medium (4 6) & $53.02 \pm 9.90$ & & $31.31 \pm 3.43$ & $(<.001)$ & $29.73 \pm 3.42$ & $(<.001)$ & $27.80 \pm 3.82$ & $(.808)$ & $89.92 \pm 9.58$ & (< \\
\hline & High (7 10) & $65.94 \pm 7.16$ & & $37.91 \pm 3.71$ & - & $24.70 \pm 3.93$ & & $27.28 \pm 5.03$ & & $102.66 \pm 13.69$ & \\
\hline
\end{tabular}

${ }^{*} p<.05 ; * \star p<.01 ; * \star * p<.001 ;$ Scheffé test $=a, b, c, d$

Correlations among resilience, professional quality of life, and degree of care

Pearson's correlation coefficients were calculated to analyse the correlation among resilience, professional quality of life, and degree of caring behavior (Table 4). Between resilience and the subdomains of professional quality of life, there was a significant strong correlation with compassion satisfaction ( $r=.80$, $\mathrm{p}<.001$ ), showing that the higher the resilience, the higher the compassion satisfaction. In addition, between resilience and the subdomains of professional quality of life, burnout ( $r=-.68, p<.001)$ showed a significant inverse correlation of relatively high strength, indicating that burnout decreased as resilience increased. Among the subdomain of the professional quality of life, compassion satisfaction showed a significant inverse correlation with burnout ( $r=-.70$, $\mathrm{p}<$.001). As compassion satisfaction increased, burnout decreased, however burnout and secondary traumatic stress $(r=.34, p<.001)$ showed a significant positive but weak correlation, indicating that the higher the burnout, the higher the secondary traumatic stress. Furthermore, the degree of caring behavior showed a significant positive correlation with resilience $(r=.43, p<.001)$ and compassion satisfaction $(r=.51, p<.001)$. These results indicated that the higher the satisfaction, the higher the degree of caring behavior, and further showed a significant inverse correlation with the burnout ( $r=-.42, p<.001)$, indicating that the lower the degree of burnout, the higher the degree of caring behavior (Table 5).

Table 5

Correlations among Resilience, Professional Quality of Life and Caring ( $\mathrm{N}=107)$

\begin{tabular}{|c|c|c|c|c|c|}
\hline \multirow[t]{2}{*}{ Variable } & \multirow[t]{2}{*}{ Resilience } & \multicolumn{3}{|l|}{ Professional quality of life } & \multirow[t]{2}{*}{ Caring } \\
\hline & & Compassion satisfaction & Burnout & Secondary traumatic stress & \\
\hline Professional quality of life & 1.00 & & & & \\
\hline Compassion satisfaction &  & 1.00 & & & \\
\hline Bumout & $-.68^{\star \star \star}$ & 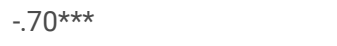 & 1.00 & & \\
\hline Secondary traumatic stress & .16 & 0.93 & 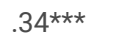 & 1.00 & \\
\hline Caring & $.43^{\star \star \star}$ & $.51^{\star \star \star}$ & $-.42^{\star \star \star}$ & .07 & 1.00 \\
\hline
\end{tabular}

The factors affecting Caring

For the multiple regression analysis using the stepwise method. As independent variables, resilience and three subdomains of professional quality of life were selected which had a statistically significant correlation with the nurses' degree of caring behavior. As a result of the univariate analysis, education and job satisfaction with a statistically significant difference from nurses' degree of caring behavior were included. Education, the nominal variable, was converted to a dummy variable and analysed. As a result of verifying the variance expansion factor and correlation to confirm the multicollinearity between the independent variables, the variance inflation factor (VIF) of caring was 1.01 2.74, which was less than 10, confirming that there was no correlation between the independent variables. Additionally, the Durbin-Watson value was 1.62, within the range of 1.5 to 2.5 which satisfies the assumption of independence between the independent variables and can admit the suitability of the regression model analysis. The final regression model set in this study was found to be statistically significant $(F=26.48, p<.001)$. Through the stepwise multiple regression analysis, it was confirmed that the factors affecting nurses' degree of caring behavior were compassion satisfaction $(t=6.00, p<.001)$ and education above graduate school $(t=3.45, p=.001)$. The degree of caring behavior showed a significant strong correlation with compassion satisfaction and education above graduate school, and it was found that it also increased when compassion satisfaction or education above graduate school increased. This model explained the degree of caring behavior by 32.5\% (Adjusted R2) (Table 6). 
Table 6

Factors affecting oncology nurses' caring

\begin{tabular}{|llllllll|}
\hline Predictor Variable & b & SE & B & t & p & Adj $\mathbf{R}^{2}$ & F(p) \\
\hline Constant & 55.61 & 6.43 & & 8.65 & $<.001$ & .325 & 26.48 \\
& & & & & & & $(<.001)$ \\
\hline Compassion satisfaction & 1.14 & .19 & .48 & 6.00 & $<.001$ & & \\
\hline Higher than graduate school & 11.29 & 3.28 & .28 & 3.45 & .001 & & \\
\hline Adj $\mathrm{R}^{2}=$ Adjusted $\mathrm{R}^{2}$ & & & & & & & \\
\hline
\end{tabular}

\section{Discussion}

This study aimed to provide basic data for interventions to improve caring for oncology nurses by investigating the resilience, professional quality of life, and degree of caring behavior of oncology nurses and the factors affecting nurses' degree of caring behavior.

In the regression model derived from the results of this study, it can be observed that it is difficult to fully understand caring, as it only explained $32.5 \%$ to the variance of caring. Among the factors influencing the degree of caring behavior of the study participants, compassion satisfaction ( $t=6.00, p<.001)$ and education above graduate school $(t=3.45, p=.001)$ were significant among the professional quality of life of the nurses. It could be estimated that the degree of caring behavior will increase if they receive graduate school, or higher, education. This estimation is supported by results from other research [26], which found that a person with high compassion satisfaction tends to show eagerness to enter school or that compassion satisfaction increases through higher education. In addition, oncology nurses' caring defined in this study means holistic nursing, which includes physical, emotional, and spiritual nursing. Interestingly, education above graduate school may be thought to have increased the degree of caring behavior, owing to learning about integrated nursing, including compassion satisfaction and physical nursing, which are the basics of holistic nursing. Although resilience, professional quality of life, and the degree of caring behavior had statistically significant correlations with each other in the correlation analysis, the regression analysis was insufficient to explain caring. In order to improve the caring for oncology nurses, a wide range of studies will be needed to elucidate other variables or parameters that are associated with resilience, professional quality of life, and caring.

The resilience of the nurse participants was slightly lower than that of general ward nurses (Averge mean 2.33 \pm 0.46 ), intensive care unit nurses (Averge mean $2.41 \pm 0.47$ ), and operating room nurses (Mean $75.9 \pm 11$ ), using the same instrument $[16,27,28]$. This relates to our finding that $75.7 \%$ of the nurses in this study showed higher burnout than average. We found the significantly negative relationship between burnout and resilience has been demonstrated in other study for general nurses [29]. According to them, we may think that high burnout of the nurse participants related to low resilience. It can assume that the reason that the oncology nurses' burnout is higher than that of nurses in other specialties is due to the characteristic of oncology nursing. In other study, the American and Canadian oncology nurses also presented high levels of burnout and compassion fatigue [30]. Perhaps it can be predicted that oncology nursing is because the nurses tend to sacrifice their personal and psychological needs to meet the patient's needs more prominently [30].

As for the professional quality of life, $75.7 \%$ of the nurse participants showed moderate or higher burnout, $83.1 \%$ showed moderate or higher secondary traumatic stress, and $26.2 \%$ showed high compassion satisfaction. Similarly, in other studies, job stress and burnout in oncology nurses were higher than in general ward nurses and nurses in other specialities $[11,20]$. In other study of the professional quality of life of oncology nurses in the United States and Canada, oncology nurses in the United States reported high compassion satisfaction (58\%), low burnout (52\%), and low secondary traumatic stress (52\%). In addition, Canadian oncology nurses reported high compassion satisfaction (59\%), low burnout (54\%), and low secondary traumatic stress (52\%) [30]. As a result, we founded that there was a difference from this study. It can be expected that the age and marital status of the study participants had an effect on their professional quality of life. $67.3 \%$ and $75.7 \%$ of the participants in this study were under 30 years of age and unmarried. In contrast, only $13 \%$ and $5 \%$ of study participants in the United States and Canada were under 30 , and $18 \%$ and $14 \%$ were unmarried. This is because, in this study, we founded that compassion satisfaction and burnout, which are subdomain of professional quality of life, were related to age and marital status. Therefore, it will be necessary to develop an intervention to improve the professional quality of life, targeting unmarried oncology nurses under the age of 30 who are at risk of declining professional quality of life.

We also found that the two variables, compassion satisfaction and burnout in the participants, was low when there is a lack of supporting resources. In addition, our study showed that when the total clinical experience was between 1 and 3 years, working in three shifts, particularly in the ward, as a staff nurse with lower job satisfaction, would generate higher levels of burnout and secondary traumatic stress. Research participants with 1 to 3 years of clinical experience may experience more burnout and lower degree of caring behavior. Benner [31] said in 'From novice to expert' that a nurse with 2 to 3 years of clinical experience can perform accurate nursing, but the risk of burnout is high, and the nursing outcome may be low. This group is consistent with what we studied as a group that needs a lot of support. Therefore, mentors assigned at hospitals, for nurses with less than 3 years of clinical experience or those lacking supporting resources, or implementing an intervention program that enhances compassion satisfaction. can reduce negative consequences such as resign.

In the present study, $5.61 \%$ of the nurses showed high burnout, secondary traumatic stress, and low compassion satisfaction. The average of resilience and degree of caring behavior of this group was lower than that of all participants, and the professional quality of life was found to have an effect on resilience and degree of caring behavior. Therefore, finding high-risk groups through regular professional quality of life measurements and participating in intervention programs could be considered as another way to improve the degree of caring behavior. 
The results of this study showed that job satisfaction was also significantly related to resilience, professional quality of life, and caring. Therefore, in order to increase the resilience of oncology nurses, who show higher burnout than nurses in other specialities and generally have low resilience, interventions should be considered necessary to increase job satisfaction by lowering burnout, leading to an increased degree of care. In order to perform proper interventions, it will be necessary to study the factors affecting job satisfaction and implementation.

The average caring score of the nurse participants in this study was 4.76 points, which was lower than the average level of care 5.27 reported in general hospital nurses, measured using the same tool in the other study [32]. It is possible that a low score was obtained because the hospital to which this study's participants belong is a tertiary hospital. Tertiary hospital is where the severity of patients in need of physical care is prioritized based on the acute stage of the disease. So holistic nursing is important, we found that oncology nursing, which is currently important in this study's clinical settings, is centred on physical nursing. This can be inferred through the fact that the education received by the nurse participants who answered yes to the question regarding whether they had ever received education related to cancer patients was education on physical care such as chemotherapy or coping with the side effects of cancer treatment. Nevertheless, since the degree of caring behavior measured in this study assesses physical, mental, and spiritual care as a whole, it seems that the degree of caring behavior in this study centered on physical nursing may have been low. Therefore, it is necessary to emphasize the importance of holistic nursing and encourage the practice of emotional and spiritual nursing, in addition to physical nursing.

\section{Limitations of the study}

Since the resilience defined in this study measures only individual factors, excluding organizational factors, there were limitations in understanding the resilience of nurses. Therefore, a research study including not only individual factors but also organizational factors is necessary. Furthermore, since the regression model obtained in this study only explains $32.5 \%$ of care, it will be useful to explore the remaining unexamined variables and to find other parameters.

\section{Conclusions}

This study found that the oncology nurse's resilience, professional quality of life, and degree of caring behavior influence each other, and factors that influence these characteristics were identified. We demonstrated that oncology nurses' caring relates to resilience, professional quality of life, and education. In order to increase oncology nurses' caring, it will be necessary to study the factors affecting nurses' compassion satisfaction, and to try to promote compassion satisfaction according to the study. It is also suggested to conduct research on additionally needed education in the graduate school to increase caring. Oncology nurses with 1 to 3 years of total clinical experience or without a support system can be a high-risk group with high burnout and low resilience, and low professional quality of life. Therefore, oncology nurses with these characteristics should be selected in advance and, if necessary, intervention programs should be developed to present coping strategies to these nurses. In addition, adequate emphasis on the importance of mental and spiritual nursing as well as physical nursing in tertiary hospital must be encouraged as a practice. This study provides oncology nurses with the importance of mental and spiritual nursing as well as physical nursing, professional quality of life that enables a positive coping system, such as resilience, and subjective quality assessment of nurses' jobs. There is an applied significance which is expected to be helpful in applying holistic nursing to cancer patients in the future and providing practical guidelines for the nursing practice.

\section{Abbreviations}

Nurses: Registered nurses

\section{Declarations}

\section{Availability of data and materials}

Additional data files in Korean are available upon request to the corresponding author.

\section{Acknowledgements}

This article is based on part of the first author's master thesis. The authors thank the participating oncology nurses who volunteered and participated in this study.

\section{Funding}

No funding was received for this study. All data collection and analysis fees were paid by the authors.

\section{Ethics declarations}

\section{Ethics approval and consent to participate}

Prior to the study, approval of the Korea University Medical Center Institutional Review Board (IRB NO: KUGH15023) was obtained and all study procedures adhered to the principles. The study followed the Declaration of Helsinki. Written informed consent was obtained from all the triage nurses. All methods were performed in accordance with the relevant guidelines and regulations. 


\section{Consent for publication}

Consent for publication was received from the participates.

\section{Competing interests}

The author(s) declared no potential conflicts of interest concerning the research, authorship, and/or publication of this article.

\section{References}

1. Lee MS, Kim HJ, Suh SR. Uncertainty and nursing need according to illness phases in cancer patient J Korean Acad Soc Nurs Edu 2008;20(1):1-9, Korean

2. Ekedahl M, Wengström Y. Nurses in cancer care-stress when encountering existential issues Eur J Oncol Nurs 2007;11(3):228-37, https://doi.org/10.1016/j.ejon.2006.09.005

3. Potter P, Deshields T, Berger JA, Clarke M, Olsen S, Chen L. Evaluation of a compassion fatigue resiliency program for oncology nurses Oncol Nurs Forum 2013;40(2):180-7, https://doi.org/10.1188/13.0NF.180-187

4. Slocum-Gori S, Hemsworth D, Chan WW, Carson A, Kazanjian A. Understanding compassion satisfaction, compassion fatigue and burnout: A survey of the hospice palliative care workforce Palliat Med 2011;27(2):172-8, https://doi.org/10.1177/0269216311431311

5. Kim HS. A comparative study regarding health condition and work stress of nurses working in cancer ward and general ward $\mathrm{J}$ Korean Oncol Nurs 2001;1(2):191-203, Korean

6. Lee YS, Tae YS. The lived experience of the burnout of nurses working in oncology wards J Korean Oncol Nurs 2012;12(1):100-9, https://doi.org/10.5388/aon.2012.12.1.100

7. Choi JS, Park S. Comparison of job stress, hardness, and burnout of nurses between advanced general hospitals and general hospitals The Journal of the Korea Contents Association 2012;12(3):251-9, https://doi.org/10.5392/JKCA.2012.12.03.251

8. Toh SG, Ang E, Devi MK. Systematic review on the relationship between the nursing shortage and job satisfaction, stress and burnout levels among nurses in oncology/haematology settings Int J Evid Based Healthc 2012;10(2):126-41, https://doi.org/10.1111/j.1744-1609.2012.00271.x

9. Yi YJ, Lee B. Research trend of nurses' job stress: A comparative study Korean J Occup Health Nurs 2013;22(1):13-23, https://doi.org/10.5807/kjohn.2013.22.1.13

10. Kim JS, Kim GS, Choi M, Lee H. Factors associated with burnout for nurses working in hematology-oncology wards Korean J Occup Heal Nurs 2011;20(1):24-34, https://doi.org/10.5807/kjohn.2011.20.1.024

11. Demerouti E, Bakker AB, Nachreiner F, Schaufeli WB. A model of burnout and life satisfaction amongst nurses J Adv Nurs 2000;32(2):454-64, https://doi.org/10.1046/j.1365-2648.2000.01496.x

12. Lu H, Barriball KL, Zhang X, While AE. Job satisfaction among hospital nurses revisited: A systematic review Int J Nurs Stud 2012;49(8):1017-38, https://doi.org/10.1016/j.ijnurstu.2011.11.009

13. Van Bogaert P, Kowalski C, Weeks SM, Van heusden D, Clarke SP. The relationship between nurse practice environment, nurse work characteristics, burnout and job outcome and quality of nursing care: A cross-sectional survey Int J Nurs Stud 2013;50(12):1667-77, https://doi.org/10.1016/j.jinurstu.2013.05.010

14. Folkman S, Lazarus RS, Gruen RJ, Delongis A. Appraisal, coping, health status, and psychological symptoms J Pers Soc Psychol 1986;50(3):571-9, https://doi.org/10.1037/0022-3514.50.3.571

15. Gillespie BM, Chaboyer W, Wallis M, Grimbeek P. Resilience in the operating room: developing and testing of a resilience model J Adv Nurs 2007;59(4):427-38, https://doi.org/10.1111/j.1365-2648.2007.04340.x

16. Moon IO, Park SK, Jung JM. Effects of resilience on work engagement and burnout of clinical nurses J korean Acad Nurs Adm 2013;19(4):525-35, https://doi.org/10.11111/jkana.2013.19.4.525

17. Cho HJ, Jung MS. Effect of empathy, resilience, self-care on compassion fatigue in oncology nurses J korean Acad Nurs Adm 2014;20(4):373-82, https://doi.org/10.11111/jkana.2014.20.4.373

18. Shang DJ, Friese DCR, Wu ME, Aiken LH. Nursing practice environment and outcomes for oncology nursing Cancer Nurs 2013;36(3):206-12, https://doi.org/10.1097/NCC.0b013e31825e4293

19. Isaac T, Zaslavsky AM, Cleary PD, Landon BE. The relationship between patients' perception of care and measures of hospital quality and safety Health Serv Res 2010;45(4):1024-40, https://doi.org/10.1111/j.1475-6773.2010.01122.x

20. Sinclair HA, Hamill C. Does vicarious traumatisation affect oncology nurses? A literature review Eur J Oncol Nurs 2007;11(4):348-56, https://doi.org/10.1016/j.ejon.2007.02.007

21. Connor, K. M., Davidson, J. R. Development of a new resilience scale: the Connor-Davidson Resilience Scale (CD-RISC) Depression and anxiety 2003;18(2): 76-82, https://doi.org/10.1002/da.10113

22. Baek HS, Lee KU, Joo EJ, Lee MY, Choi KS. Reliability and validity of the Korean version of the Connor-Davidson resilience scale Psychiatry Investig 2010;7(2):109-15, https://doi.org/10.4306/pi.2010.7.2.109

23. Stamm BH Professional quality of life elements theory and measurement [Internet]; 2012. Available from: https://proqol.org/.

24. Nelson J, Watson J. Measuring caring: International research on caritas as healing Springer Publishing Com New York.2012, pp. 436. 
25. Cha ES, Kim KH, Erlen JA. Translation of scales in cross-cultural research: Issues and techniques J Adv Nurs 2007;58(4):386-95, https://doi.org/10.1111/j.1365-2648.2007.04242.x

26. Kim S, Kim JH, Park JY, Suh EY, Yang HJ, Lee Sy et al. Oncology nurses' professional quality of life in a tertiary hospital J Korean Clin Nurs Res 2010;16(3):145-55, Korean

27. Kim SN, Yoo MS. Relationships between resilience, job stress, and organizational commitment in ICU nurses J Korean Acad Soc Home Care Nurs 2014;21(1):36-43, Korean

28. Kim BN, Oh HS, Park YS. A study of nurses' resilience, occupational stress and satisfaction Korean J Occup Heal Nurs 2011;20(1):14-23, https://doi.org/10.5807/kjohn.2011.20.1.014

29. Guo, Y-f, Luo, Y-h, Lam, L, Cross, W, Plummer, V, Zhang, J-p. Burnout and its association with resilience in nurses: A cross-sectional study J Clin Nurs. 2018; 27: 441-449, https://doi.org/10.1111/jocn.13952

30. Wu, S., Singh-Carlson, S., Odell, A., Reynolds, G., Su, Y. Compassion fatigue, burnout, and compassion satisfaction among oncology nurses in the United States and Canada Oncol Nurs Forum 2016;43(4):161-69, https://doi.org/10.1188/16.0NF.E161-E169

31. Benner. From novice to expert Am J Nurs 1982;82(3):402-7, https://doi.org/10.2307/3462928

32. Clerico, E., Harley, C. Caring for the Nurse in the Hospital Environment International Journal of Human Caring 2013;17(1):56-63, https://doi.org/10.20467/1091-5710.17.1.56 